\title{
2009 Trends in Small-Volume Dissolution Apparatus for Low-Dose Compounds
}

\author{
G. Bryan Crist \\ Varian, Inc., 13000 Weston Parkway, Cary, NC 27513
}

e-mail:Bryan.Crist@Varianinc.com

\begin{abstract}
The concept of small-volume dissolution arises from the need to determine the dissolution rate from low-dose, generally high potency drugs or drugs with analytical techniques that are not very sensitive. Novel drug delivery products including subcutaneous implants, ocular systems, microspheres, nanospheres, and combination products such as drug-eluting stents (DES) have demanded more from traditional dissolution and drug-release apparatus. To maintain quantitative levels of analyte during the dissolution test, a reduction in vessel volume accompanied by an alteration of compendial dissolution apparatus may be required (1). The utilization of small-volume dissolution apparatus stems from the need to provide accurate, reliable data for decision-making during drug development stages and assurance of quality when the formulation reached full-scale production, as well as to maintain future assurance of product quality and stability. This topic will explore the use and general limitations of typical compendial USP apparatus, noncompendial modifications to the standard USP apparatus, and non-USP apparatus designed to yield small-volume dissolution methods with reliable results. In addition, related issues concerning apparatus calibration, method development, and method validation of the modified dissolution apparatus will be reviewed.
\end{abstract}

\section{INTRODUCTION}

$$
\text { T. }
$$
he two components of the dissolution test are simple: sample preparation, which takes place within the dissolution apparatus, and sample analysis, primarily via chromatographic or spectrophotometric techniques. The two components of the dissolution test, sample preparation and assay, are separated by a filtration step. Filters must be validated to prove their efficiency in removing undissolved active pharmaceutical ingredient (API) from the sample and to verify that they do not adsorb dissolved API, which affects the integrity of the sample concentration. The first step taken to overcome concentration and sensitivity issues is usually modification of the analytical method by taking advantage of a larger path length for a spectrophotometer or a larger injection volume for high performance liquid chromatography (HPLC). When success with these techniques is limited, we must focus on the dissolution apparatus where a reduction in media volume may be a more rugged solution.

Only a few of the compendial dissolution and drug-release apparatus are designed for low-dose compounds and volume requirements less than $100 \mathrm{~mL}$ present additional challenges to the traditional paddle and basket apparatus.

Rugged dissolution methods should quantify the low levels of analyte accurately and precisely, especially in the initial stages of drug release in the dissolution profile. Accurate analytical concentration in the presence of reliable and consistent agitation is the primary requirement of any dissolution test but demands extreme precision in low-volume conditions to ensure data accuracy. The dissolution apparatus must operate under conditions of controlled temperature, agitation rate, precise hydrodynamics, and volume. To maintain precise hydrodynamics, the apparatus must maintain overall physical uniformity and alignment throughout the test. Standard dissolution apparatus may be obtained from manufactures that produce the equipment according to the specification and tolerances outlined in General Test Chapter $<711>$ Dissolution (2).

For low concentrations of API, official compendial apparatus may be incapable of maintaining quantitative levels of analyte during the dissolution of oral dosage units containing microgram or nanogram levels.

Dissolution of typical high-potency, low-dose compounds may require a reduction in vessel volume accompanied by an alteration in apparatus design due to limitations in detection and quantitation. If a reduction in volume is considered and the apparatus is modified, the operating conditions of the modified apparatus should maintain the same degree of precision and alignment required for any other compendial dissolution apparatus.

Small-volume apparatus are desired not only for high-potency, low-dose formulations. Small-volume dissolution utilizing a mini paddle has been suggested as an alternative to standard paddle methods that require large volumes of biorelevant medium. This can be very expensive, and there may be limitations of large sample size or active drug availability in the early stages of drug development (3).

In the current regulatory environment, our dissolution methods must be accurate, sensitive, and specific, and the reproducibility of the test method employed must be established, verified, and documented (4). Additionally, the method must maintain limits of detection and quantitation, range, and linearity and discriminate variation from batch to batch. Small-volume apparatus components should be precisely designed, rugged, and 
commercially available to aid in the approval of the noncompendial method and analytical transferability.

Regulatory expectations of the small-volume

dissolution method should continue to:

- Characterize the in vitro release early in development.

- Evaluate release with various conditions of agitation, media composition, $\mathrm{pH}$, and temperature.

- Establish optimum test conditions.

- Demonstrate a release of $80 \%$ or an asymptote.

The method should also be capable of showing discrimination, rejecting lots, and ultimately demonstrating consistency of performance from lot to lot. In terms of dissolution specifications for modified release products, expectations are high for in vitro-in vivo correlation (IVIVC), and the small-volume dissolution apparatus should provide data needed to support scale-up and post-approval changes for modified-release (SUPAC-MR) (5). Additionally, the dissolution test should be approvable based on meaningful methodology and specifications, and in general, the method must be relevant, predictable, specific, and discernable (6).

\section{DISCUSSION OF DISSOLUTION APPARATUS}

Over the years, the traditional paddle and basket dissolution apparatus with 1-L vessels has been an important tool for characterizing the biopharmaceutical quality of a product at different stages in the product life cycle. Our discussion will focus on those official apparatus contained in the current USP $(2,7)$.

Apparatus mentioned in the following sections contain recognized minimum operational volumes. Consultation with the manufacturers of these apparatus is recommended to obtain the latest performance specifications if further reductions in vessel volume are desired. Apparatus will be discussed according to their USP apparatus numbers, and additional information will follow each apparatus regarding its noncompendial modifications to achieve small-volume dissolution.

\section{USP Apparatus 1 and 2-Rotating Basket and Rotating Paddle}

The traditional basket and paddle apparatus utilize a 1000-mL hemispheric bottom vessel. To accomplish sink conditions required for bolus and poorly soluble dosage forms, vessels up to $4000 \mathrm{~mL}$ have been incorporated into the USP, but the operational minimum of the $1000-\mathrm{mL}$ vessel is around $500 \mathrm{~mL}$. Although smaller volumes have been used for various purposes such as the dissolution of esomeprazole magnesium and omeprazole magnesium, which are carried out in the acid stage using only $300 \mathrm{~mL}$ of $0.1 \mathrm{M} \mathrm{HCl}(8)$, in general, the hydrodynamics below $500 \mathrm{~mL}$ become too unstable for routine dissolution testing. The traditional vessel has handled routine oral dosage tablets, capsules, suspensions, suppositories, and chewable tablets, but smaller-volume vessels with scaled-down basket and paddles (Figure 1) have been developed to handle volumes capable of nano- and picogram levels of drug.

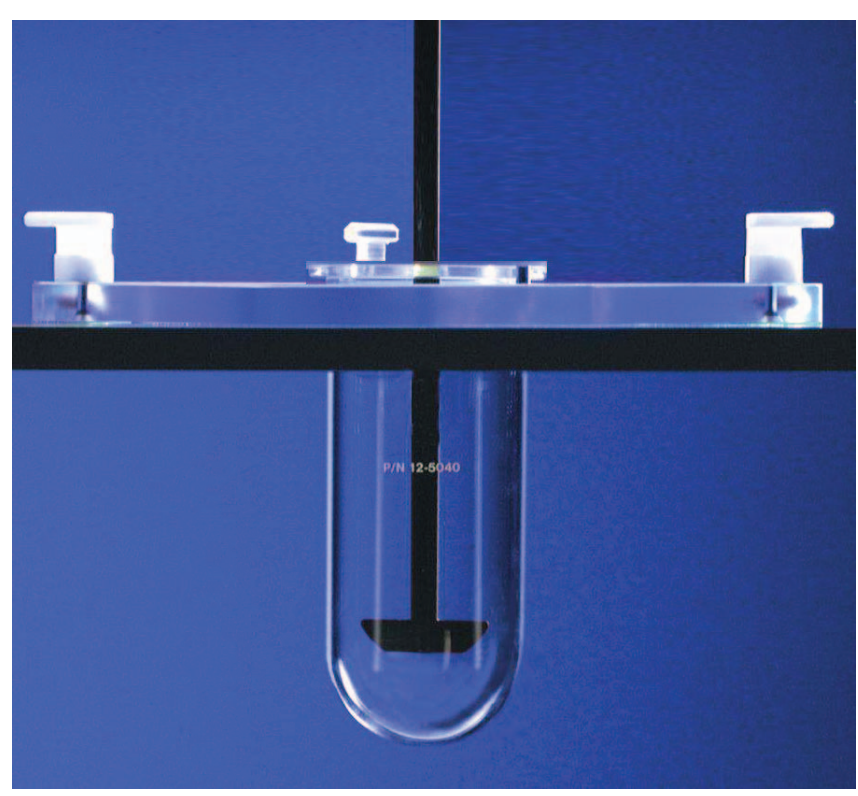

Figure 1. 100-mL small-volume dissolution vessel and mini paddle.

The operational minimum for small-volume vessels of $100 \mathrm{~mL}$ and $200 \mathrm{~mL}$ is approximately $30 \mathrm{~mL}$.

\section{USP Apparatus 3-Reciprocating Cylinder}

The reciprocating cylinder apparatus has six or seven inner tubes, which mechanically traverse six rows of corresponding media-filled outer tubes. Also called the Bio-Dis, for biorelevant dissolution, the apparatus has been successfully used for tablets, capsules, beads (Figure 2), and other extended-release dosage forms that require exposure to various media representing conditions in the gastrointestinal tract. The traditional configuration utilizes a $300-\mathrm{mL}$ vessel, which is an advantage for products requiring a small volume. The reciprocating cylinder has an operational minimum of about $150 \mathrm{~mL}$. A noncompendial version has been developed; it utilizes a $100-\mathrm{mL}$ vessel with a scaled-down reciprocating inner tube that can run at an operational minimum of about $50 \mathrm{~mL}$.

\section{USP Apparatus 4-Flow-Through Cell}

The flow-through cell was originally developed to simulate gastrointestinal conditions by exposing extended-release and poorly soluble dosage forms to media of varying $\mathrm{pH}$. The apparatus has been used for capsules, powders, tablets, implants, and suppositories and has been used with a wide range of media volume. A compendial closed system with a small media reservoir could traditionally reduce volume to less than $100 \mathrm{~mL}$ for the official USP 12- and 26-mm cells. Noncompendial cells that allow dissolution of nanoparticles and suppositories have been developed. Small-volume applications have been refined for the flow-through apparatus, resulting in closed cells handling dissolution volumes less than $15 \mathrm{~mL}$. 


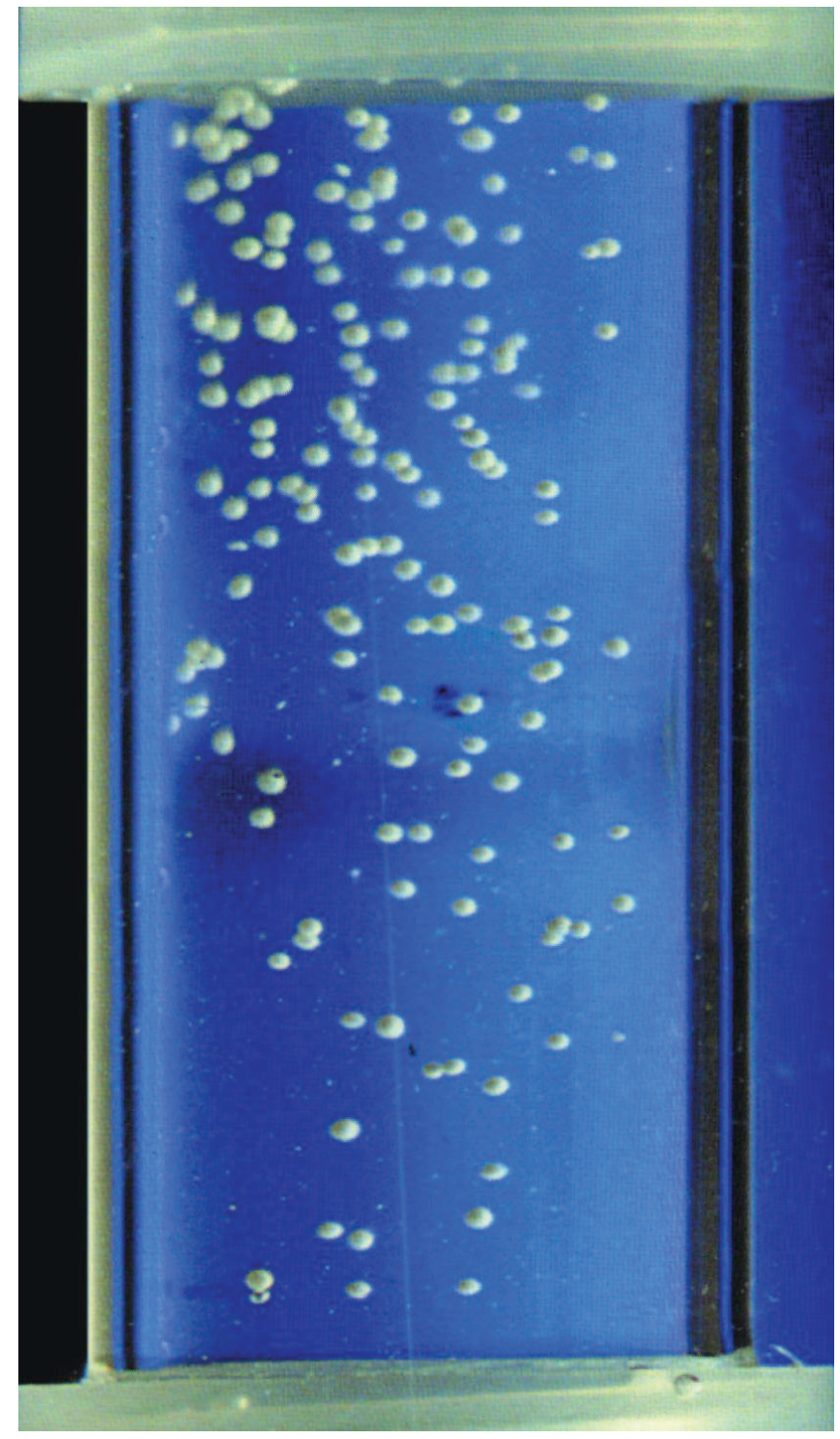

Figure 2. Theophylline beads in reciprocating cylinder apparatus.

These smaller cells have been developed for implants and other low-dose products.

\section{USP Apparatus 5 and 6-Paddle Over Disk and Rotating Cylinder}

These two methods were developed for transdermal systems, and the official vessel is the traditional $1000-\mathrm{mL}$ hemispheric bottom dissolution vessel mentioned previously. The minimum vessel volume is $500 \mathrm{~mL}$ due to the hydrodynamic issue mentioned for the paddle apparatus. No smaller vessels have been developed or applied to USP Apparatus 5 or 6 to the author's knowledge at this time.

\section{USP Apparatus 7-Reciprocating Holder}

Originally introduced in the USP as a small-volume option for small transdermal patches, the reciprocating disk apparatus was later renamed the reciprocating holder apparatus with the adoption of four additional holders for transdermal systems, osmotic pumps, and other low-dose delivery systems. The agitation rate for the reciprocating holder apparatus has been typically 30 cycles per minute at an amplitude of $2 \mathrm{~cm}$, but a specific vessel size has not been stated in the USP General Chapter <724> Drug Release (7). The original reciprocating holder apparatus commonly used $50-400-\mathrm{mL}$ vessels and was not initially designed for extremely low volumes. The reciprocating cylinder apparatus has become a good candidate for modification due to the emergence of numerous low-dose compounds that have challenged traditional dissolution equipment. These dosage forms include subcutaneous implants and combination products such as drug-eluting stents (Figure 3). Currently, Apparatus 7 can accommodate a dissolution environment as low as $5 \mathrm{~mL}$.

\section{Non-USP Apparatus}

An early drug release apparatus that allowed a dosage form to be exposed to various media in a rotating bottle apparatus appeared in The National Formulary in 1975 (9). This apparatus, while quite labor intensive, provided a small-volume dissolution environment with the unique advantage of no evaporative loss; media and the dosage form were contained in a sealed $150-\mathrm{mL}$ tube that rotated within a water bath. This apparatus has never become one of the official dissolution apparatus although it is still used for some implants and dosage forms that may take weeks or months to dissolve. Volumes as low as $3 \mathrm{~mL}$ have been achieved by introducing implants into 4-mL HPLC vials, which are placed in small holders on the rotating bottle apparatus.

Microvolume dissolution environments have been commercially produced with fiber-optic probes placed in several milliliters to evaluate the dissolution of API or powders. Other efforts to provide a dissolution environment have been attempted, but controlling temperature and agitation rates was difficult. Some of

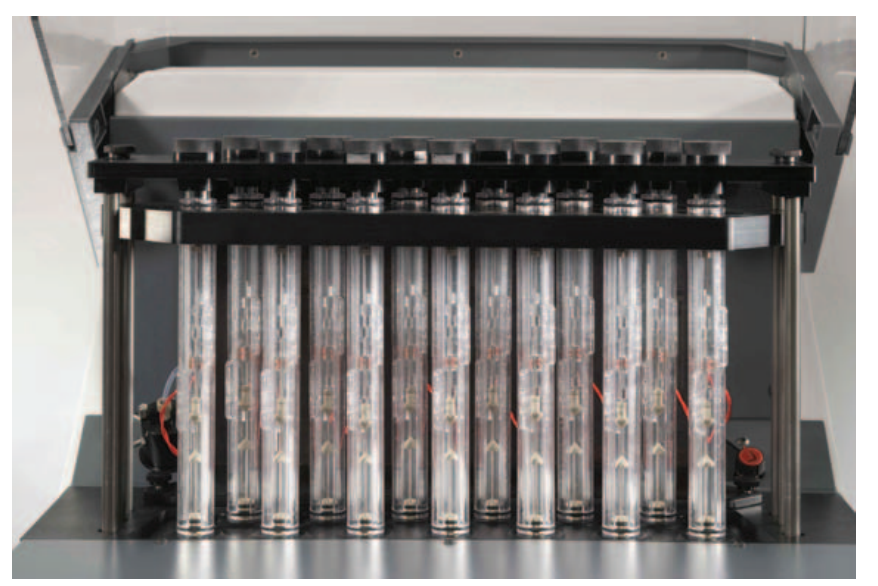

Figure 3. Small-volume Apparatus 7 with reciprocating holders for drug-eluting stents. 
these apparatus have involved shaker tables within an incubator or rotary mixers contained in temperature-controlled incubators. For some dosage forms, dissolution in such apparatus may lead to inconsistencies in dissolution rate due to battering and rough handling of the dosage form during the test.

\section{Challenges for Small-Volume Dissolution Apparatus}

As you can see, many apparatus have been modified in some way to provide smaller testing volumes. As modifications are applied, physical parameters critical to the alignment of the dissolution apparatus along with volumetric accuracy have to be maintained. As with traditional dissolution equipment, agitation rate, centering, wobble, verticality, height settings, and temperature control or any other critical parameter must be routinely measured and documented. This provides essential assurance of the mechanical integrity of a modified system that may not benefit from a performance verification program using actual product similar to the USP Performance Verification Tests (PVT) with prednisone and salicylic acid, which are designed for use in 1-Liter dissolution vessels. Commercially available small-volume apparatus and equipment should be used wherever possible, and the manufacturer specifications and tolerances should be used as the basis for mechanical verification of components. Modified apparatus must also ensure that the dissolution environment is isolated from the effects of vibration, drafts, or other circumstances that may affect the accuracy of the dissolution test.

The road to the justification of small-volume dissolution apparatus is not always an easy one. While the opportunity to utilize a small-volume noncompendial dissolution apparatus may be obvious, justification of its use is warranted through a sometimes exhaustive process of elimination. Standard compendial dissolution apparatus should be used as a first choice, but failures resulting from improper sensitivity, correlation, or biorelevance may warrant the use of small-volume apparatus, which may be accomplished through modifications to existing apparatus. For dissolution volumes less than $500 \mathrm{~mL}$, UV cells with increased path lengths up to $5 \mathrm{~cm}$, if feasible, or HPLC analysis with relatively large injection volumes to justify that limits and quantitation cannot be met with traditional dissolution apparatus should be considered. It may also be feasible to explore alternate analytical techniques including fluorescence and mass-spectrometry. This is especially important at early time points for timed-release or extended-release formulations because the concentrations should be well beyond limits of quantitation for necessary profiling of the drug release.

Dissolution methodology must maintain parameters of accuracy, precision, limit of detection, limit of quantitation, linearity, robustness, and transferability that can be validated, and small-volume dissolution is no exception. During the drug development process, critical decisions are made based on analytical data that may be acquired with small-volume apparatus. This makes it imperative that dissolution equipment is properly qualified and methods are properly validated.

In summary, small-volume dissolution apparatus should not contribute to or produce unreliable data that was characteristic of early dissolution apparatus. The two essential components of the dissolution test, sample preparation and assay, must be properly evaluated and validated separately. The greatest value in validating a rugged small-volume dissolution apparatus and its associated analytical method is not in the fulfillment of regulatory requirements, but in providing accurate, reliable data for decision making during drug development stages and assurance of quality when the product reaches full-scale production.

\section{REFERENCES}

1. Palmieri, A., Ed. Dissolution Theory, Methodology, and Testing; Dissolution Technologies, Inc.: Hockessin, DE, 2007.

2. Dissolution $<711>$. In United States Pharmacopeia and National Formulary USP 31-NF 26; The United States Pharmacopeial Convention, Inc.: Rockville, MD, 2008.

3. Klein, S. The Mini Paddle Apparatus-a Useful Tool in the Early Development Stage? Experiences with Immediate-Release Dosage Forms. Dissolution Technol. 2006, 13 (4), 6-11.

4. Testing and release for distribution. Current Good Manufacturing Practice for Finished Pharmaceuticals, Code of Federal Regulations, Title 21, Vol. 4, Part 211.165(e), 2008

5. Scale-Up and Postapproval Changes for Modified Release Solid Oral Dosage Forms; Guidance for Industry; U.S. Department of Health and Human Services, Food and Drug Administration, Center for Drug Evaluation and Research (CDER), U.S. Government Printing Office:Washington, DC, 1997.

6. Mehta, M. FDA Expectations. Consortium on Drug Eluting Stents; Office of Pharmaceutical Science, Food and Drug Administration, Center for Drug Evaluation and Research (CDER); March 25, 2003.

7. Drug Release $<724>$. In United States Pharmacopeia and National Formulary USP 31-NF 26; The United States Pharmacopeial Convention, Inc.: Rockville, MD, 2008; Vol. 1.

8. Dissolution Methods for Drug Products Database, 2008. U.S. Department of Health and Human Services, Food and Drug Administration, Center for Drug Evaluation and Research (CDER) Web site. http://www.accessdata.fda.gov/scripts/cder/ dissolution/dsp_SearchResults_Dissolutions. cfm?PrintAll=1 (accessed Jan 15, 2009).

9. Timed-Release Tablets and Capsules-In Vitro Test Procedure. In National Formulary XIV; The United States Pharmacopeial Convention, Inc.: Rockville, MD, 1975. 